\title{
Combined structural and spectroscopic investigations of a series of photo- and thermoswitchable trinitrocobalt(III) coordination compounds in the solid state
}

\author{
Krystyna A. Deresz, Sylwia E. Kutniewska, Radosław Kamiński, Katarzyna N. Jarzembska \\ Chemistry Department, University of Warsaw, Żwirki i Wigury 101, Warsaw, Poland \\ krystynaderesz@gmail.com
}

Photocrystallography is a technique used to determine light-induced structural changes accompanying formation of excited-state species, or other photoreaction products, in crystals via X-ray diffraction methods. Among others, it can be employed to investigate photo-linkage isomerism in the solid state. Photoswitchable compounds of this kind contain ambidentate ligands, e.g., $\mathrm{NO}_{2}, \mathrm{SCN}$ or NO groups, which can bind to a metal centre in several different ways. Such linkage isomers can exhibit different physical properties such as: colour, density, or conductivity, which makes them interesting materials for various applications. Photoswitchable systems can be used in data storage devices, solar panels, as biological markers, etc.

The current project is dedicated to comprehensive investigations of a series of trinitrocobalt(III) coordination compounds [1] which are potential photoswitchable systems. According to literature cobalt nitrocomplexes may exhibit thermoswitchable properties [2], but there is in general very limited information about light-induced linkage isomerism of this group of compounds. In turn, three nitro groups attached to a metal centre interact in a crystal structure in different ways, which enables analyses of the crystal packing effect on photoswitchable properties. In the course of our research conditions assuring the most effective isomerisation reaction in the examined crystal systems were determined on the basis of multi-temperature X-ray diffraction, photocrystallographic and spectroscopic measurements results. Additionally, the influence of packing and intermolecular interactions on the isomerisation reaction was examined. The experimental findings were supported by theoretical computations.

It appeared that some of the studied compounds undergo the nitro group isomerisation reaction along with temperature changes, while the metastable-state form, nitrito isomer, may exist up to a relatively high temperature (about $240 \mathrm{~K}$ ). Nevertheless, the thermoinduced conversion usually does not exceed 30\%. UV-Vis-light irradiation (230-660 nm) of the crystal samples elevates the observed conversion by only several percent. It was observed that the nitro groups engaged in relatively strong hydrogen bonds with the amine fragments of adjacent molecules do not undergo any transformation. Also, the stabilisation of the potential metastable-state form in the crystal structure is of great importance.
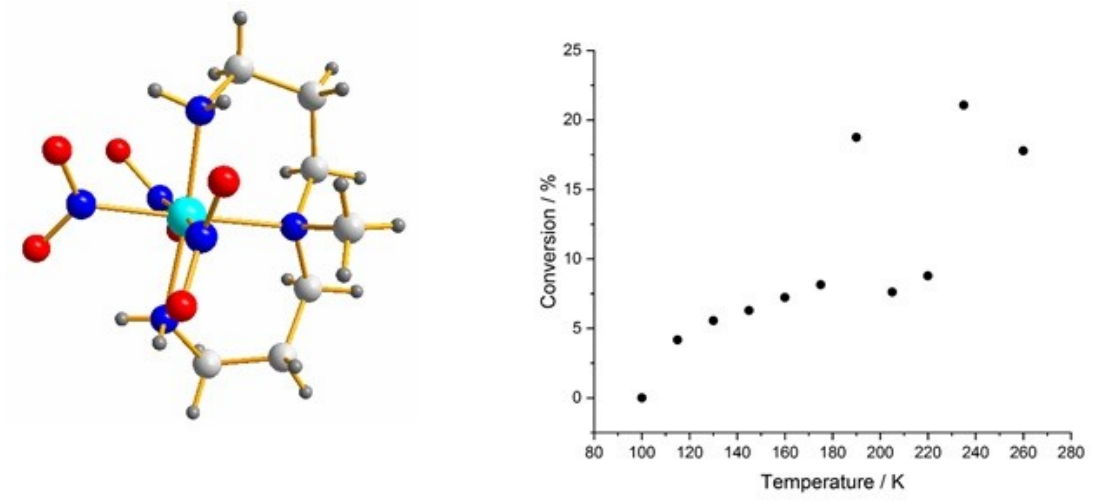

Figure 1. An example chemical compound with its conversion during multitemperature measurements

[1] Chun H.; Bernal I. (2001) Crystal Growth \& Design 1, 67-72

[2] Granthe I.; Nordin E. (1979) Inorganic Chemistry 18, 1109-1116

Keywords: photocrystallography; coordination compounds; nitro group isomerisation

The authors thank A. Krówczyński and W. Buchowicz (Warsaw, Poland) for assistance during the syntheses of the examined compounds. The PRELUDIUM grant (2017/25/N/ST4/02440) of the NCN (Poland) is gratefully acknowledged for financial support. The X-ray diffraction experiments were carried out at the Department of Physics, UW, on a Rigaku Oxford Diffraction SuperNova diffractometer, which was co-financed by the EU within the European Regional Development Fund (POIG.02.01.00-14-122/09). 